\section{Fortgeschrittenes GIST: Imatinib verlängert PFS}

\author{
Eine adjuvante Imatinib-Therapie verlängert das rezidivfreie Überleben \\ von Patienten mit fortgeschrittenem GIST. Nun wurden langfristige Vor- \\ und eventuelle Nachteile einer 2-Jahres-Medikation überprüft.
}

V or über 10 Jahren wurde Imatinib zur Behandlung fortgeschrittener gastrointestinaler Stromatumoren (GIST) eingeführt. 2004 startete eine Phase-III-Studie, an der 908 Patienten mit GIST und hohem oder intermediärem Risiko nach einer R0- oder R1-Resektion teilnahmen. Untersucht wurde die Wirksamkeit einer 2-jährigen Behandlung mit $400 \mathrm{mg} / \mathrm{d}$ Imatinib im Vergleich zu keiner adjuvanten Behandlung. Primärer Endpunkt war zunächst das Gesamtüberleben (OS). Angesichts der deutlich verbesserten Überlebenschancen der Patienten wurde 2009 als neuer primärer Endpunkt das Imatinib-versagensfreie Überleben (IFFS) im- plementiert, d.h. die Zeit bis zu einer Resistenzentwicklung gegen die Substanz. 2012 erfolgte eine Interimsanalyse mit 835 Patienten unter Berücksichtigung des neuen primären Endpunkts. Nach median 4,7 Jahren ergab sich eine Rate für das 5 -Jahres-IFFS von $87 \%$ in der Imatinibversus $84 \%$ in der Kontrollgruppe (Hazard Ratio [HR] 0,79; $p=0,21$ ). Für das rezidivfreie Überleben (RFS) lag die Rate nach 3 Jahren bei 84 versus $66 \%$ und nach 5 Jahren bei 69 versus $63 \%$ (log-rank $\mathrm{p}<0,001)$. Die Rate für das 5-Jahres-OS betrug 100 versus $99 \%$. Bei den 528 Patienten mit Hochrisiko-GIST ergab sich eine Rate für das 5-Jahres-IFFS 79 vs.
$73 \%$, bei Diagnose durch eine zentrale Pathologie 77 versus $73 \%$.

Fazit: Bei Patienten mit Risiko-GIST verlängern 2 Jahre Imatinib adjuvant das RFS. Beim IFFS ergab sich kein Unterschied gegenüber keiner ImatinibTherapie. Womöglich sind jedoch neue Strategien mit z. B. kombinierter Gabe mehrerer zielgerichteter Agenzien und eine optimierte Medikationsdauer effektiver als die vor 10 Jahren noch hochinnovative 2-jährige Imatinib-Therapie.

Barbara Kreutzkamp

Casali PG et al. Time to definite failure to first tyrosine kinase inhibitor in localized GI stromal tumors treated with imatinib as an adjuvant: a European Organisation for Research and Treatment of Cancer Soft Tissue and Bone Sarcoma Group Intergroup randomized trial in collaboration with the Australasian Gastro-Intestinal Trials Group, UNICANCER, French Sarcoma Group, Italian Sarcoma Group, and Spanish Group for Research on Sarcomas. J Clin Oncol. 2015;33(36):4276-83.

\title{
Überlebensvorteil durch TKI bei Hochrisiko-GIST
}

\section{Infolge der ersten Auswertung einer skandinavisch-deutschen Studie wird Patienten mit gastrointestinalen Stromatumoren (GIST) und hohem Risiko nach der Tumorresektion eine 3-jährige Therapie mit Imatinib empfohlen. Jetzt liegt eine geplante zweite Analyse mit längerer Beobachtungszeit vor.}

Z wischen 2004 und 2008 wurden für die gemeinsame Studie der Skandinavischen Sarkom Gruppe XVIII und der Arbeitsgemeinschaft Internistische

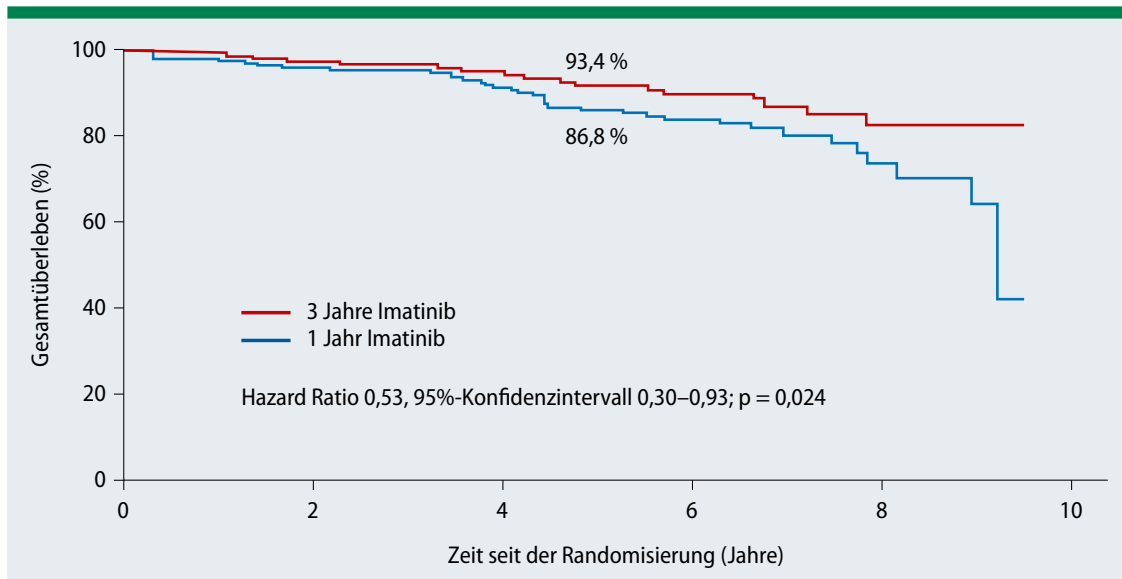

Abb. 1: Besonders die „efficacy population“ profitierte von einer längeren Imatinib-Gabe.
Onkologie (AIO) 400 Patienten mit makroskopisch komplett entferntem, KITpositivem GIST und hohem Rezidivrisiko rekrutiert. Nach der Resektion erhiel-

ten die Patienten randomisiert entweder 1 oder 3 Jahre Imatinib (400 mg/d).

Nach median 90 Monaten betrug die 5-Jahres-Rate für das rezidivfreie Überleben (RFS) bei 3-jähriger ImatinibTherapie $71,1 \%$, bei 1-jähriger ImatinibGabe 52,3\% (Hazard Ratio [HR] 0,60; $\mathrm{p}<0,001)$, Die 5-Jahres-Gesamtüberlebensrate betrug 91,9 versus $85,3 \%$ (HR $0,60 ; \mathrm{p}=0,036)$. Besonders deutlich profitierten Patienten der „efficacy population“, deren GIST-Diagnose zentral bestätigt worden war und die keine makroskopischen Metastasen zum Zeitpunkt der Randomisierung aufgewiesen hatten (93,4 vs. 86,8\%; HR 0,53; $\mathrm{p}=0,024 ;$ Abb. 1).

Fazit: Bei einem resezierbaren GIST ist nach der Operation die Einnahme von Imatinib über 3 Jahre mit einem Überlebensvorteil im Vergleich zu einer 1-jährigen Therapie verbunden. Die erreichten 5-Jahres-Überlebensraten sind hoch. Inzwischen wird auch eine noch längere adjuvante Imatinibgabe in Studien untersucht. Friederike Klein 